\title{
Purification of clavulanic acid produced by Streptomyces clavuligerus via submerged fermentation using polyethylene glycol/cholinium chloride aqueous two-phase systems
}

\author{
Paweł Panas ${ }^{\text {a, b }}$, Camila Lopes ${ }^{\text {a }}$, Marcel O. Cerri ${ }^{a}$, Sónia P.M. Ventura ${ }^{\text {, }}$, \\ Valéria C. Santos-Ebinuma ${ }^{\text {, }}$, Jorge F.B. Pereira ${ }^{\text {a, * }}$ \\ ${ }^{a}$ Department of Bioprocess and Biotechnology, School of Pharmaceutical Sciences, UNESP - Univ Estadual Paulista, Rodovia Araraquara-Jaú/01, Campos \\ Ville, 14800-903, Araraquara, SP, Brazil \\ b Institute of Botany, Leibniz Universität Hannover, Herrenhäuser Str. 2, 30419 Hannover, Germany \\ ${ }^{\text {c } C I C E C O}$ - Aveiro Institute of Materials, Department of Chemistry, University of Aveiro, 3810-193 Aveiro, Portugal
}

\section{A R T I C L E I N F O}

\section{Article history:}

Received 16 May 2017

Received in revised form

10 July 2017

Accepted 11 July 2017

Available online 12 July 2017

\section{Keywords:}

Clavulanic acid

Aqueous two-phase systems

Polyethylene glycol

Cholinium chloride

Streptomyces clavuligerus

\begin{abstract}
A B S T R A C T
Clavulanic acid (CA) is an important pharmaceutical compound produced by batch fermentation of Streptomyces clavuligerus. Since, CA is chemically unstable, its downstream processing should be studied to develop more efficient and resolute techniques. Herein, the use of aqueous two-phase systems (ATPS) composed of cholinium chloride, $[\mathrm{Ch}] \mathrm{Cl}$, was proposed as a novel platform for the recovery and purification of CA. Thus, the stability of CA in presence of different $[\mathrm{Ch}] \mathrm{Cl}$ concentrations was initially studied, and the high biocompatibility of this salt was demonstrated by the low CA degradation levels. Then, the partitioning of CA using two types of polymeric ATPS has been investigated. Two ATPS formed by the combination of $[\mathrm{Ch}] \mathrm{Cl}$ and two polyethylene glycol (PEG) polymers, PEG $600 \mathrm{~g} \mathrm{~mol}^{-1}$ (PEG-600) and polyethylene glycol methyl ether $550 \mathrm{~g} \mathrm{~mol}^{-1}$ (PEG-500-OMe), were used to assess the influence of the PEG nature, in addition to the concentration of the phase forming agents on the CA partitioning. It has shown that $\mathrm{CA}$ is almost equally distributed between the two-phases in equilibrium $\left(0.6<K_{C A}<1.6\right)$. Nevertheless, the selective extraction of CA for the [Ch]Cl-rich or PEG-rich phase by the proper adjustment of ATPS composition was attained. In the search for higher extraction efficiencies [EE (\%)] and partition coefficients $\left(K_{C A}\right)$, a second polymeric ATPS platform composed of PEG-600 and sodium polyacrylate $8000 \mathrm{~g} \mathrm{~mol}^{-1}$ (NaPA-8000), applying [Ch]Cl as adjuvant was tested. The main results suggest the recovery of $C A$ towards the PEG-rich phase $\left(K_{C A} \geq 5.6 \pm 0.6\right.$ and $\left.E E \geq 85.5 \pm 1.4 \%\right)$. The higher migration levels of CA have mainly resulted from the electronegative repulsion of NaPA-8000 over CA molecules. The ATPS with best performance for the CA extraction were selected for the recovery of CA directly from fermented broth of Streptomyces clavuligerus. In this set of experiments, the highest values of CA recovery yield and purification factor (respectively, $64.91 \pm 1.99 \%$ and $22.70 \pm 0.87$ ) were attained for the systems PEG-600/NaPA-8000 and PEG-600/[Ch]Cl, respectively.
\end{abstract}

() 2017 Elsevier B.V. All rights reserved.

\section{Introduction}

Clavulanic acid (CA) is an organic compound which acts as a $\beta$ lactamase inhibitor, presenting a broad-spectrum activity against Gram-positive and Gram-negative bacteria [1]. It is widely used in medicine as potassium salt in combination with other biologically active compounds, such as antibiotics from the penicillin and

\footnotetext{
* Corresponding author.

E-mail address: jfbpereira@fcfar.unesp.br (J.F.B. Pereira).
}

amoxicillin groups [2]. There are some studies pointing to additional activity of CA, namely those representing some modulation effects in the central nervous system [3].

At industrial scale, CA is produced by batch fermentation using mainly Streptomyces clavuligerus and its extraction and purification is considered as a complex and multistep downstream process. This common process starts with the clarification of the medium by filtration or centrifugation, followed by a further purification step, normally involving an adsorption or liquid-liquid extraction step, which commonly requires the use of potentially hazardous organic 
solvents. Finally, the final purification stage is based on the combination of different chromatographic techniques [4-7]. It is wellknown that CA is chemically unstable due to its structure. Thus, to keep its stability this compound is mostly maintained in the form of sodium, potassium or lithium salts. Even so the extraction yields achieved nowadays are generally quite low making CA production cost-intensive $[2,5,8,9]$. All these limitations have been pushing the development or improvement of alternative, cheaper and more environmental friendly purification methods to improve the efficiency of CA recovery and to decrease the process costs.

In the search for improved CA downstream processes aqueous two-phase systems (ATPS; also, called aqueous biphasic systems, ABS) have been point out as a promising and biocompatible alternative. This technique is used to separate and recover macromolecules and has already been successfully applied on the separation of enzymes, alkaloids, antibiotics and biopharmaceuticals [10-16]. ATPS traditionally consist on the combination of two distinct watersoluble components as phase forming agents, e.g. polymerpolymer, polymer-salt or salt-salt [10]. After separation, the mixture will create two immiscible phases: a lighter top one and a heavier bottom one. The phase forming agents differ in their chemical and physical properties, but together and above certain concentration conditions these can form an effective separation system [10]. Depending of the mixture composition, and mainly when used for the recovery of biomolecules from complex fermented media, an interface (commonly defined as a third layer) containing some or the main contaminants can be also formed, which is recognized in literature as the formation of three phase partitioning systems (TPP) [17]. This interesting feature allows the application of ATPS as an effective purification process even at an industrial scale. However, it is worth to mention that precipitation at the interface occurs at quite low protein concentrations [18].

A high number of ATPS have been reported, most of them combining inorganic salts and polyethylene glycol (PEG) polymers as phase forming agents $[6,11,15,19,20]$. However, due to the character of conventional high-melting inorganic salts applied on the ATPS formation some of these systems have been facing some industrial environmental concerns, in particular, salts crystallization problems or eutrophication potential of phosphate salts $[10,21]$. Recently, following the interest for more biocompatible and biodegradable salts, the use of cholinium salts as effective phase formers was also proposed; yet, these systems are not significantly studied [11,22-28]. Choline was recognized by the Institute of Medicine as an essential nutrient, due to its main role in human body (such as, neurotransmitter synthesis, cell-membrane signaling and structuring) [29], and considered as an important component for several biological functions, like the synthesis of folic acid and vitamin $B_{12}$ [30]. Cholinium-based salts are constituted by the 2-hydroxyethyl-N,N,N-trimethylammonium as common cation, which could be combined with a wide range of distinct anions, such as chloride, acetate, malate, etc., by a simple and cheap acid-base reaction. Among these salts, cholinium chloride $([\mathrm{Ch}] \mathrm{Cl})$ is one of the most interesting, since it is highly hygroscopic, an important additive in animal feed (in particular, for chicken feed) and is massively produced at an industrial scale [31]. Despite of its high melting point $\left(302{ }^{\circ} \mathrm{C}\right),[\mathrm{Ch}] \mathrm{Cl}$ shows low toxicity and high biodegrability [32-36], which makes it a perfect candidate for several biotechnological applications. In this context, $[\mathrm{Ch}] \mathrm{Cl}$ can be a feasible option to promote the formation of ATPS when combined with PEG [37]. The use of PEG polymers as co-phase forming agents re-enforces the biocompatible character of these systems. Actually, these belong to one of the principal groups of polymers most widely used in chemical and life-science industries. Their environmentally friendly character, high biodegradability, low volatility, high water solubility and low cost $[38,39]$ are thus making them very good candidates to be used in the ATPS formation.

However, the formation of polymeric ATPS is not happening exclusively by the addition and/or combination of polymers and salts. In the last decade, the formation of polymeric ATPS composed of sodium polyacrylate (NaPA) and PEG was also reported $[40,41]$. These PEG/NaPA-based ATPS were applied as effective platforms for the biocompatible extraction of several biomolecules from fermented broth, namely proteins [42], and clavulanic acid [6]. The "bio"-stability characteristics of these systems are not only a result of PEG properties already highlighted, but also from the harmless character of NaPA [6].

Although some ATPS were already used for the extraction of CA from fermented broth $[4,6,7,43-45]$ showing satisfactory results in terms of recovery rates and partition coefficients, their application at industrial scale has facing some serious problems, namely regarding their environmentally unfriendly character and high cost of the overall process, mainly related with the phase formers cost. In addition, most of these studies have not fully explained the separation mechanisms of CA. Thus, considering the benefits of using $[\mathrm{Ch}] \mathrm{Cl}$ and biocompatible polymers like PEG and NaPA as ATPS two-phase formers, this work has evaluated the extraction and recovery of CA directly from the fermented broth of $S$. clavuligerus using [Ch]Cl-based ATPS, here proposed as alternative, sustainable and novel CA purification' platform. Actually, this type of ATPS platforms is considered as more efficient and sustainable, since $[\mathrm{Ch}] \mathrm{Cl}$ is an essential nutrient precursor of vitamin synthesis (B complex and thiamine) and is also part of the head groups of cell membrane phospholipids; derived from natural sources and it is considered as non-toxic for example for Vibrio fischeri bacteria [35]. In order to accomplish this task, firstly the partitioning of commercial CA in three distinct series of ATPS was investigated, namely those combining aqueous solutions of $[\mathrm{Ch}] \mathrm{Cl}$ and three different polymers. The applicability of these systems was evaluated by means of the partition coefficients and extraction efficiencies of commercial CA achieved. In a second stage, the most performant ATPS were selected and further evaluated for the direct recovery and purification of CA from the fermented broth of $S$. clavuligerus.

\section{Experimental section}

\subsection{Materials}

Polyethylene glycol with average molecular weight of $600 \mathrm{~g} \mathrm{~mol}^{-1}$ (PEG-600), polyethylene glycol methyl ether with average molecular weight of $550 \mathrm{~g} \mathrm{~mol}^{-1}$ (PEG-500-OMe), sodium polyacrylate with average molecular weight of $8000 \mathrm{~g} \mathrm{~mol}^{-1}$ (NaPA-8000, 45 wt\% in water) and cholinium chloride ([Ch]Cl, with purity $\geq 99 \mathrm{wt} \%$ ) were provided by Sigma-Aldrich Brazil, Ltda. The remaining reagents are of analytical grade and were used as received. Ultrapure water double distilled, passed through a reserve-osmosis system, and further treated by filtration through a Millipore Milli-Q ion-exchange system was used.

Two distinct samples of clavulanic acid (CA) were used in the experiments, a commercial one obtained from the commercial drug Clavulin $^{\circledR}$ (500 mg of amoxicillin and $125 \mathrm{mg}$ of potassium clavulanate) from GlaxoSmithKline (West Sussex, England) and one produced via fermentation by Streptomyces clavuligerus ATCC 27064. In a first set of experiments, the commercial CA was used, aiming at the optimization of the partition processing conditions. With this purpose, one pill of commercial CA was crushed in a mill and dissolved in pure water. After filtration, the clarified solution was moved into a $1000 \mathrm{~mL}$ flask and the volume was filled up to $500 \mathrm{~mL}$. For all experiments, a final concentration of CA around $300-400 \mathrm{mg} \mathrm{L}^{-1}$ was used. The calibration curve was established based on pure potassium clavulanate (with purity of $99.8 \mathrm{wt} \%$ ) from 
Sigma-Aldrich Brazil, Ltda. In the second set of experiments, samples of CA produced by S. clavuligerus ATCC 27064 via fermentation (for more details see Section 2.2) were used. The detailed information about the materials mentioned above is listed in Table 1.

\subsection{Microorganism maintenance and fermented processes}

The S. clavuligerus ATCC 27064 was kept frozen at $-70{ }^{\circ} \mathrm{C}$ in $10 \%$ $(\mathrm{v} / \mathrm{v})$ glycerol in cryotubes. After thawing, vegetative cell suspensions kept in cryotubes $(3.5 \mathrm{~mL})$ were inoculated into $25 \mathrm{~mL}$ of the reactivation medium in an Erlenmeyer flask (total volume of $250 \mathrm{~mL}$ ). The suspension was then incubated in an orbital shaker at $28^{\circ} \mathrm{C}, 250 \mathrm{rpm}$ for $24 \mathrm{~h}$. After the incubation, $2.5 \mathrm{~mL}$ of the bacterial suspension were diluted in $22.5 \mathrm{~mL}$ of fermentation medium and incubated on an orbital shaker under the same conditions. Afterwards, $5.0 \mathrm{~mL}$ of cell suspension were added to $45 \mathrm{~mL}$ of fresh fermentation medium in an Erlenmeyer flask $(500 \mathrm{~mL})$ and incubated on an orbital shaker under the same conditions for $72 \mathrm{~h}$. After fermentation, the broth was centrifuged at $3720 \times \mathrm{g}$ for $15 \mathrm{~min}$ at $5{ }^{\circ} \mathrm{C}$ in a centrifuge, model Hettich - Universal 320R. The supernatant obtained from this process was characterized and it was achieved $630 \mathrm{mg} \mathrm{L}^{-1}$ (quantified as described in Section 2.4.4) of CA at $\mathrm{pH}$ 6.8. The supernatant was recovered and stored at $-80^{\circ} \mathrm{C}$ for further studies.

\subsection{Media composition}

The seed medium used presented the following composition (in $\mathrm{g} \mathrm{L}^{-1}$ distilled water): glycerol, 15.0; bacto peptone, 10.0; malt extract, 10.0; yeast extract, $1.0 ; \mathrm{K}_{2} \mathrm{HPO}_{4}, 2.5 ; \mathrm{MgSO}_{4} \cdot 7 \mathrm{H}_{2} \mathrm{O}, 0.75$; $\mathrm{MnCl}_{2} \cdot 4 \mathrm{H}_{2} \mathrm{O}, 0.001 ; \mathrm{FeSO}_{4} \cdot 7 \mathrm{H}_{2} \mathrm{O}, 0.001 ; \mathrm{ZnSO}_{4} \cdot 7 \mathrm{H}_{2} \mathrm{O}, 0.001 ; \mathrm{MOPS}$ buffer, 21 (equivalent to $100 \mathrm{mM}$ ). The medium was adjusted to $\mathrm{pH}$ 6.8 with a $\mathrm{NaOH}(5 \mathrm{M})$ solution prior to be autoclaved at $121^{\circ} \mathrm{C}$ for $15 \mathrm{~min}$. The culture medium for the production of CA used in the present work presents the following composition (in $\mathrm{g} \mathrm{L}^{-1}$ ) [46]: glycerol, 15.0; isolated soy protein, 20.0; $\mathrm{K}_{2} \mathrm{HPO}_{4}, 0.5 ; \mathrm{MnCl}_{2} \cdot 4 \mathrm{H}_{2} \mathrm{O}$, $0.001 ; \mathrm{FeSO}_{4} \cdot 7 \mathrm{H}_{2} \mathrm{O}, 0.4 ; \mathrm{ZnSO}_{4} \cdot 7 \mathrm{H}_{2} \mathrm{O}, 0.001$, MOPS buffer, 21 ; at $\mathrm{pH}$ 6.8 .

\subsection{Methods}

\subsubsection{Clavulanic acid stability at different [Ch]Cl concentrations}

The stability of the commercial CA was checked against different concentrations of $[\mathrm{Ch}] \mathrm{Cl}$, specifically in the range of $2.15-4.3 \mathrm{M}$, at $25{ }^{\circ} \mathrm{C}$. To test its stability, a stock aqueous solution of CA (300-400 $\mathrm{mg} \mathrm{L}^{-1}$ ) was set and used in the preparation of each aqueous solution of CA (30-40 $\left.\mathrm{mg} \mathrm{L}^{-1}\right)$. Afterwards, the solutions were homogenized in an orbital mixer, at $8 \mathrm{rpm}$ for $5 \mathrm{~min}$ at $25^{\circ} \mathrm{C}$, and kept in a water bath at $25{ }^{\circ} \mathrm{C}$ (New Ethics, SP, Brazil) during $180 \mathrm{~min}$. The stability of CA was then evaluated after 60,120 and $180 \mathrm{~min}$ in equilibrium ( $180 \mathrm{~min}$ was defined as the maximum time of CA exposure, since it corresponds to the maximum time for carrying out the extraction process, results not shown). At each time, an aliquot was withdrawn and the percentage of $C A$ remaining in the $[\mathrm{Ch}] \mathrm{Cl}$ aqueous solution, or CA non-degraded [CA (\%)] was determined by Eq. (1):

$\mathrm{CA}(\%)=\frac{[\mathrm{CA}]_{\text {sol }}}{[\mathrm{CA}]_{0}}$

where $[\mathrm{CA}]_{\text {sol }}$ is the CA concentration in solution $\left(\mathrm{mg} \mathrm{L}^{-1}\right)$ after incubation and $[\mathrm{CA}]_{0}$ is the initial CA concentration (at $0 \mathrm{~min}$ ). All the experiments were carried out in triplicate and the respective standard deviations calculated.

\subsubsection{Partition of commercial $\mathrm{CA}$ in [Ch]Cl-based ATPS}

In order to evaluate the best systems and experimental conditions promoting the partition and purification of CA directly from the fermented broth, three polymeric ATPS based in [Ch]Cl conjugated with PEG-600, PEG-550-OMe, and NaPA-8000 were tested. For each system, at least six mixture compositions at the biphasic region were selected, as presented in Table S1 in Supporting Information. The ATPS components and aqueous solutions of commercial CA (at around $300-400 \mathrm{mg} \mathrm{L}^{-1}$ ) were added to different glass tubes $(15 \mathrm{~mL})$ by their weight $\left( \pm 10^{-4} \mathrm{~g}\right)$ to obtain a total mass of $5.0 \mathrm{~g}$. Afterwards, the solutions were homogenized in an orbital mixer, at $8 \mathrm{rpm}$ for $5 \mathrm{~min}$ at $25^{\circ} \mathrm{C}$, and kept in a water bath (New Ethics, SP, Brazil) during $180 \mathrm{~min}$. After the equilibrium, and guaranteeing the complete separation of the phases, the systems were centrifuged at $2000 \times g$ for $15 \mathrm{~min}$. The respective volumes of the co-existing phases were visually identified and determined by using the glass tubes scale. Then, both top and bottom phases were carefully separated using Pasteur-pipettes, and the CA concentration ( $\mathrm{mg} \mathrm{L}^{-1}$ ) determined. All the assays were performed in triplicate, and the respective standard deviations determined.

The partitioning behavior in each series of ATPS was assessed in terms of CA partition coefficient, $K_{C A}$, and CA extraction efficiency for the PEG-rich phase, EE (\%), both determined according to Eqs. (2) and (3), respectively:

$K_{C A}=\frac{[\mathrm{CA}]_{\text {PEG-rich }}}{[\mathrm{CA}]_{\mathrm{PEG}-\text { poor }}}$

$$
\begin{aligned}
E E(\%)= & \frac{\mathrm{V}_{\mathrm{PEG}-\text { rich }} \times[\mathrm{CA}]_{\mathrm{PEG}-\text { rich }}}{\mathrm{V}_{\mathrm{PEG}-\text { rich }} \times[\mathrm{CA}]_{\mathrm{PEG}-\text { rich }}+\mathrm{V}_{\mathrm{PEG}-\text { poor }} \times[\mathrm{CA}]_{\mathrm{PEG}-\text { poor }}} \\
& \times 100
\end{aligned}
$$

where [CA] and $V$ are, respectively, the CA concentration $\left(\mathrm{mg} \mathrm{L}^{-1}\right)$ and the volume of the phase (L), while the subscripts PEG-rich and PEG-poor refer to the PEG-rich phase and PEG-poor phase $([\mathrm{Ch}] \mathrm{Cl}-$ rich phase or NaPA-8000-rich phase), respectively.

Note that for the systems composed of PEG-600/[Ch]Cl and PEG$550-\mathrm{OMe} /[\mathrm{Ch}] \mathrm{Cl}$, it was observed a change in the densities of the

Table 1

\begin{tabular}{|c|c|c|c|}
\hline Chemical name & Source & $\begin{array}{l}\text { Purity } \\
\text { (mass fraction) }\end{array}$ & Purification Protocol \\
\hline Polyethylene glycol average $M_{n} 600$ & Sigma-Aldrich & - & None \\
\hline Poly(ethylene glycol) methyl ether average $M_{n} 550$ & Sigma-Aldrich & - & None \\
\hline Poly(acrylic acid, sodium salt) solution average Mw 8000 & Sigma-Aldrich & - & None \\
\hline Choline chloride & Sigma-Aldrich & $\geq 99 \%$ & None \\
\hline Water & - & - & Reserve-osmosis/Filtration through a Millipore Milli-Q ion-exchange system \\
\hline Clavulin ${ }^{\circledR}$ & GlaxoSmithKline & - & Filtration \\
\hline Potassium clavulanate & Sigma-Aldrich & $\geq 99.8 \%$ & None \\
\hline
\end{tabular}

Sources and purity of the materials used in the work. 
co-existing phases, depending on the polymer/salt composition, and consequently, an inversion of the top and bottom phases nature was identified. On other hand, in the PEG-600/NaPA-8000 ATPS, the top-phase is the PEG-rich phase, while the bottom phase is a NaPA-8000-rich phase. The corresponding phases in equilibrium for all systems were determined by conductivity measurements at $25{ }^{\circ} \mathrm{C}$. All experimental data are presented in Table S1 in Supporting Information.

\subsubsection{Recovery of CA from the fermented broth}

The [Ch]Cl-based ATPS with high extraction efficiencies were chosen for the recovery of CA from the fermented broth of $S$. clavuligerus. The partition studies were performed, according to the procedure described in section 2.4.2, adding the liquid supernatant of the fermented broth and the corresponding ATPS components (polymer(s) and $[\mathrm{Ch}] \mathrm{Cl}$ ) in $15 \mathrm{~mL}$ glass tubes. Considering that at this stage, the CA is recovered from a complex fermented media (with a huge amount of interferences and contaminants), the CA recovery was evaluated by means of CA recovery yield, $\eta_{C A}(\%)$, and the purification factor, $P F$, those determined through Eqs. (4) and (5), respectively.

$\eta_{C A}(\%)=\frac{[\mathrm{CA}]_{C A-\text { rich phase }} \cdot V_{C A-\text { rich phase }}}{[\mathrm{CA}]_{i} \cdot V_{i}} \times 100$

$P F=\frac{[\mathrm{CA}]_{C A-\text { rich phase }}}{[\mathrm{TP}]_{C A-\text { rich phase }}} / \frac{[\mathrm{CA}]_{i}}{[\mathrm{TP}]_{i}}$

Where [CA] and [TP] corresponds to the concentration of CA and total protein $\left(\mathrm{mg} \mathrm{L}^{-1}\right)$, while the subscripts $C A$-rich phase represent the phase where CA was more concentrated and $i$ represents the CA initial composition (supernatant).

\subsubsection{Quantification of $C A$ and total protein content}

In order to quantify the CA a modified method by Bird et al. [47] was used. In this method, the CA was measured by the increase in the UV absorbance at $312 \mathrm{~nm}$ resulting from the release of the product [1-(4-aza-8-hydroxy-6-oxo)oct-2-en-1-oylimidazole] regarding the reaction between CA and imidazole. Briefly, $250 \mu \mathrm{L}$ of each phase (or aqueous solution) was mixed with $1 \mathrm{~mL}$ of imidazole $\left(60 \mathrm{~g} \mathrm{~L}^{-1}-\mathrm{pH} 6.8\right)$. Samples were incubated in a water bath at $35^{\circ} \mathrm{C}$ for $15 \mathrm{~min}$. The absorbance was measured at $312 \mathrm{~nm}$ in a glass cuvette using the UV spectrophotometer (Ultrospec 2100 pro, Amersham Biosciences, USA), and the corresponding CA concentration determined using a calibration curve previously established.

In order to determine the TP content and calculate the respective $F P$, a spectrofluorometric method (using Spectrofluorophotometer RF-6000 SHIMADZU) was applied. A calibration curve for the TP content was determined, using bovine serum albumin (BSA) as the model proteins. Firstly, and to determine the maximum fluorescence excitation and emission wavelengths of BSA and the impact of the solvents in the fluorescence pattern, a 3D fluorescence spectrum was acquired. The maximum BSA fluorescence was attained with an excitation at $276 \mathrm{~nm}$ and emission at $336 \mathrm{~nm}$. These wavelengths were then chosen to determine the TP calibration curve at $25^{\circ} \mathrm{C}$, and consequently, to determine the TP composition of each sample. The fluorescence pattern was not significantly altered by the phase-forming agents considering the dilutions studied.

\subsubsection{Conductivity and $\mathrm{pH}$ measurements}

After the phase settling, the $\mathrm{pH}$ and conductivity measurements of ATPS co-existing phases were determined. The $\mathrm{pH}$ values $( \pm 0.01)$ of each phase were acquired in a Technal TEC-5 (Technal Equip.
Laboratórios, Brazil) pH-meter and conductivity $\left( \pm 0.01 \mu \mathrm{S} \mathrm{cm}{ }^{-1}\right)$ using a Del Lab DL-150 (Delfini IND. COM., Brazil) conductivity meter. All measurements were carried out at $25^{\circ} \mathrm{C}$.

\section{Results and discussion}

\subsection{Clavulanic acid stability in [Ch]Cl aqueous solutions}

Clavulanic acid (CA) in its crude form is known to be chemically unstable [2], and that's why it is mostly used as a potassium salt. Many factors can influence the CA stability, since its $\beta$-lactam ring can be degraded in the presence of metallic ions in water [48], different salts, $\mathrm{pH}$ values and temperature [49]. CA is more stable in neutral or slightly acidic solutions ( $\mathrm{pH}$ between 6 and 7.2), and temperatures close to $25{ }^{\circ} \mathrm{C}$ or lower [49]. However, the aqueous solutions with high salt concentration can induce a prejudicial effect on the drug stability, causing its degradability [50]. It was in this context that some experiments were carried out to investigate the effect of different [Ch]Cl aqueous solutions towards the CA stability, being the main results depicted in Fig. 1.

The results in Fig. 1 show that the concentrations of $[\mathrm{Ch}] \mathrm{Cl}$ studied do not affect significantly the stability of CA, being the maximum loss of activity defined at around $10 \%$ after $180 \mathrm{~min}$. In the range of $[\mathrm{Ch}] \mathrm{Cl}$ concentrations evaluated, the $\mathrm{pH}$ of each aqueous solution is shown to be slightly acidic $(5<\mathrm{pH}<6)$, which means that the $[\mathrm{Ch}] \mathrm{Cl}$ can control the solution' $\mathrm{pH}$, maintaining a quite favorable condition for the drug (optimal $\mathrm{pH}$ already define at around $6.0-7.2[2,48,49])$. It is known that $\mathrm{pH}$ plays a crucial role in the CA degradation process, and thus, a comparison between degradation and the $\mathrm{pH}$ of PEG- and $[\mathrm{Ch}] \mathrm{Cl}$-rich phases was performed. With the increase of $[\mathrm{Ch}] \mathrm{Cl}$ concentration, the $\mathrm{pH}$ of both co-existing phases remains stable in the range of 5-6, since this $\mathrm{pH}$ condition is close to the optimal pH (see Table S1 from Supporting Information), consequently allowing the maintenance of CA structure without significant degradation levels, thus justifying the use of [Ch]Cl-based ATPS to recover CA. On the other hand, as illustrated in Fig. 1, the addition of $[\mathrm{Ch}] \mathrm{Cl}$ at concentrations from 2.2 to $4.3 \mathrm{M}$ have induced the same level of CA degradability, suggesting that the ionic strength of the medium has no relevant influence on the rate of CA hydrolysis. However, the CA degradation is

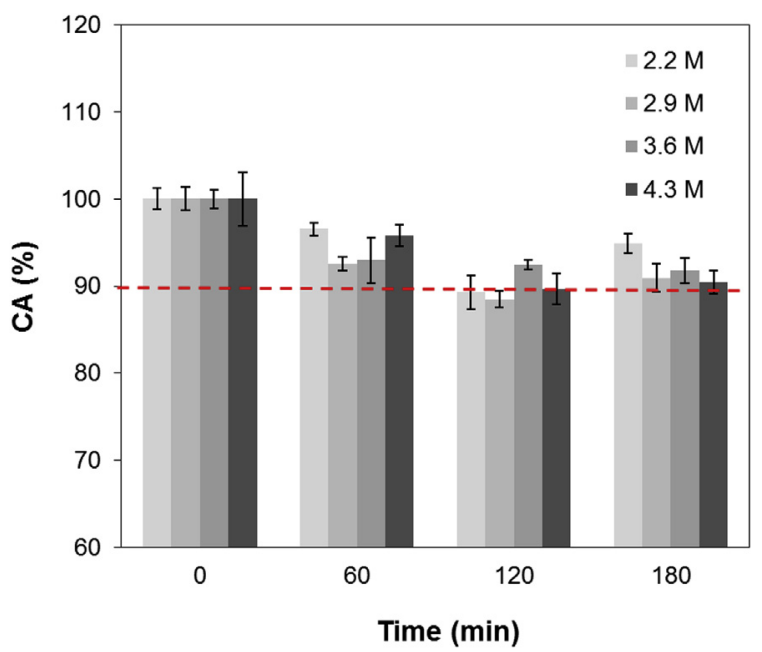

Fig. 1. Stability of $\mathrm{CA}$ in $[\mathrm{Ch}] \mathrm{Cl}$ aqueous solutions $(2.2-4.3 \mathrm{M})$ for different exposure times up to $180 \mathrm{~min}$ at $25^{\circ} \mathrm{C}$ and atmospheric pressure. Error bars represent standard deviation for three independent assays. The red line is present only as an eye guideline. (For interpretation of the references to colour in this figure legend, the reader is referred to the web version of this article.) 
more affected by the salt species than by the ionic strength [49]. Considering that $[\mathrm{Ch}] \mathrm{Cl}$ exhibits a lower ionic strength than the most common inorganic salts previously studied, the negligible influence of $[\mathrm{Ch}] \mathrm{Cl}$ ionic strength on the $\mathrm{CA}$ degradation observed in this work is easily understood. In addition, this negligible effect agrees with previous findings of Laidler [51], who highlighted that the salts' ionic strength has no relevant influence on the rate of hydrolysis reactions [51], and by Carneiro-da-Cunha et al., in which the citrate concentration effect over CA degradation was negligible [50]. These results support the high biocompatibility of $[\mathrm{Ch}] \mathrm{Cl}$, allowing the most appropriate adjustment of the $[\mathrm{Ch}] \mathrm{Cl}$ composition in each ATPS to reach the best extraction performance without compromising the CA structural integrity.

In addition, $\mathrm{CA}$ is quite stable in presence of polymeric aqueous solutions, as already demonstrated in literature for several concentrations of aqueous solutions of PEG with variable molar mass (from 2000 until $20000 \mathrm{~g} \mathrm{~mol}^{-1}$ ) [6,50] and NaPA [6]. Taking into account the CA stable character in presence of polymeric aqueous solutions, and the low CA degradability in presence of [Ch]Cl, PEG600/[Ch]Cl- PEG-550-OMe/[Ch]Cl- and PEG-600/NaPA-8000/[Ch] Cl-based ATPS were further investigated towards the partitioning and recovery of CA produced via fermentation.

\subsection{CA partitioning in ATPS composed of PEG and [Ch]Cl}

The CA partitioning using ATPS composed of different types of PEG, namely PEG-600 and PEG-550-OMe conjugated with the [Ch] $\mathrm{Cl}$ as the second main phase component, was initially assessed. Both polymers were chosen to evaluate if a proper control of the hydrogen-bond-donor ability of PEG, by replacing the terminal -OH group with an -OMe, increases the ability of the system to separate and/or concentrate the CA.

The phase diagrams of these two ATPS were previously determined [52], and it was observed a change in the densities of the coexisting phases, depending on the polymer/salt composition, and consequently, an inversion of the top and bottom phases. Thus, the corresponding phases were determined by the measurement of their conductivity at $25{ }^{\circ} \mathrm{C}$ (values presented in Table S1 from Supporting Information). To evaluate the CA partitioning capability and extraction efficiency at $25{ }^{\circ} \mathrm{C}$ considering each ATPS, five mixture points with different PEG and $[\mathrm{Ch}] \mathrm{Cl}$ compositions were prepared and the partitioning parameters determined at $25^{\circ} \mathrm{C}$, as

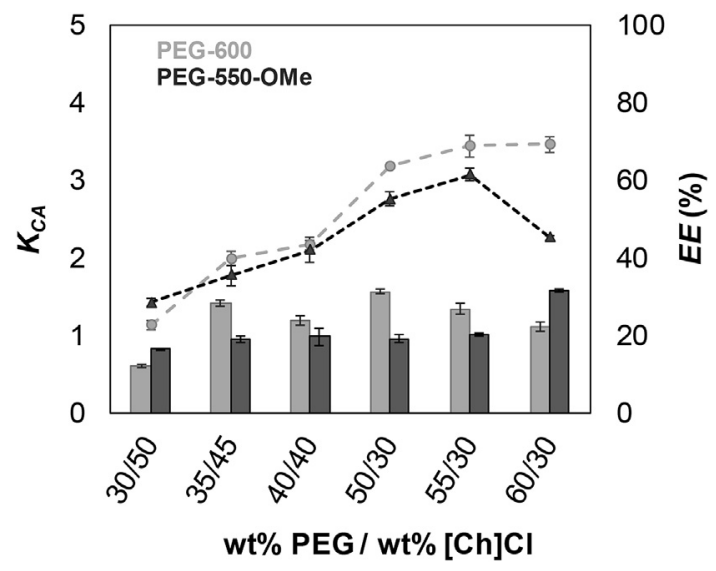

Fig. 2. Partitioning coefficients $\left(K_{C A} ;\right.$, ) and extraction efficiencies (EE (\%); , ) of CA as a function of PEG-600 or PEG-550-OMe/[Ch]Cl weight percentages of each ATPS, at $25{ }^{\circ} \mathrm{C}$ and atmospheric pressure. The light gray bars and symbols correspond to PEG600- and the dark gray symbols and bars to PEG-550-OMe-based ATPS. Error bars represent standard deviation for three independent replicates. Dashed lines are only for guideline purposes. presented in Fig. 2. The detailed average and standard deviations of the weight fraction composition (wt\%), partition coefficients of CA $\left(K_{C A}\right)$, extraction efficiencies $[E E(\%)]$ and volume ratios $\left(V_{R}\right)$ for each system are provided in Tables S1 and S2 of the Supporting Information.

The partitioning results depicted in Fig. 2 show that CA has a comparable affinity for both the co-existing phases $\left(0.6<K_{C A}<1.6\right)$. Although the CA distribution for PEG-rich or [Ch]Cl-rich phase is quite similar, in general, it is observed a slight preference of CA for the PEG-rich phase described by the results of EE (\%) and more accentuated for systems based in PEG-600 ATPS. The exception was obtained for both systems with low amounts of PEG (30 wt\%), in which the CA was concentrated in the [Ch]Cl-rich phase. These results show that the polymer content has a strong influence on the volume ratio ( $V_{R}$-values presented in Supporting Information), and consequently, in the CA extraction efficiencies. Thus, and considering that the corresponding phases' volume can be properly adjusted, it is possible to extract CA for the [Ch]Cl-rich or PEG-rich phase, just by the proper adjustment of the ATPS composition. This tailored extraction aptitude is proved through the results depicted in Fig. 2, in which the EE (\%) of CA for the PEG-rich phase varies, approximately, from 22 to $69 \%$. It is important to notice that the polymer type (PEG-600- or PEG-550-OMe) used does not affect significantly the extraction of CA, being the system based in $60 \mathrm{wt} \%$ of polymer and $30 \mathrm{wt} \%$ of $[\mathrm{Ch}] \mathrm{Cl}$ the only exception. Meanwhile, the partition coefficient data obtained in this study are lower than those previously obtained for the use of traditional PEG/inorganic salts-based ATPS [7,45,50,53].

All these previous studies have shown that CA has a high affinity towards the PEG-rich phase, which is quite hard to be explained theoretically. First of all, it is important to note that CA has a hydrophilic character $\left(\log K_{o w}=-1.23\right.$ ) [54], and considering the $\mathrm{pH}$ of most of the PEG/salt ATPS, it is mainly present in an ionic form $\left(2.3<\mathrm{pK}_{\mathrm{a}}<2.7\right)$ [7]. In addition, as CA is a small molecule (199.16 $\left.\mathrm{g} \mathrm{mol}^{-1}\right)$, it is not probably affected by exclusion volume effects of large PEG polymers. Thus, it would be expected the preferential presence of CA in the most hydrophilic phase, meaning the salt-rich phase, which is verified for most of our results (Fig. 2). In literature $[7,45,50,53]$, the high CA recoveries for the polymeric phases were not properly explained, and thus, the phenomena behind the CA partitioning were still not fully understood. Herein, CA is equally distributed by both co-existing phases, or partially recovered in the [Ch]Cl-rich phase (systems with $E E<50 \%$ ). Unlike most of the inorganic salts able to form ATPS, [Ch]Cl has a weak ability to induce the salting-out effect of other species [11,55,56], and thus, it will dehydrate the polymer-rich phase less than the common inorganic salts. Recently, we have demonstrated [57] that, although the two phases of PEG-600/[Ch]Cl-based ATPS are rich in water, the less hydrophilic environment is observed for the PEGrich phase (as shown by the water content percentages presented in Table S1 in Supporting Information). The assessment of the relative hydrophobicity of the phases in PEG-600/[Ch] Cl systems was demonstrated by the partitioning of caffeine for the lower water content PEG-rich phase, where a linear dependency between the logarithmic function of the partition coefficients and the water content ratio was found [57]. The water content ratio values presented in Table S1 (Supporting Information), demonstrate that the PEG-rich phase exhibits the most hydrophobic environment, independently of the type and composition of the system under study. Thus, considering that CA is quite hydrophilic, the relative hydrophobicity of the polymer-rich phase induces the migration of drug to the $[\mathrm{Ch}] \mathrm{Cl}$-rich phase. Similarly, and due to the high hydrophobicity of PEG-550-OMe, in comparison with PEG-600/[Ch] Cl-based ATPS, the transfer of the CA molecules towards the [Ch] $\mathrm{Cl}$-rich phase was increased, as demonstrated by the low $K_{C A}$ and 
EE values. The results obtained with both [Ch]Cl-based ATPS seem to indicate that the CA-water affinity is driven the CA partitioning in a certain extent.

\subsection{CA partitioning in ATPS composed of PEG-600 and NaPA-8000 with [Ch]Cl as adjuvant}

After the initial study with PEG/[Ch]Cl-based ATPS, a second set of experiments using commercial CA were carried in systems composed of PEG-600 and NaPA-8000, in which [Ch]Cl was only added as adjuvant. This combination PEG/NaPA has been previously reported to be an effective system for the extraction of CA from fermented broth [6], but in the previous work, the PEG/NaPA systems under study have used PEG polymers with high molecular size (higher than $2000 \mathrm{~g} \mathrm{~mol}^{-1}$ ), requiring the use of common inorganic salts as electrolytes for the two-phase formation [58]. Herein, we propose the use of a small size PEG polymer (PEG-600) as phase former in combination with NaPA-8000 polymer. These PEG-600/ NaPA-8000-based ATPS can even promote a two-phase region without the addition of electrolytes (data not shown). Thus, it was evaluated the capability of ATPS of different PEG-600/NaPA-8000 and $[\mathrm{Ch}] \mathrm{Cl}$ as adjuvant to separate the commercial CA, being evaluated the effect of PEG-600 and $[\mathrm{Ch}] \mathrm{Cl}$ concentration in the CA partitioning at $25^{\circ} \mathrm{C}$. The results of $K_{C A}$ and $E E(\%)$ of CA in each ATPS were depicted in Fig. 3. The detailed data considering mean values and respective standard deviations of partition coefficients, extraction efficiencies and compositions of each ATPS mixture point used are compiled in Table S3 of Supporting Information.

The gathered results of Fig. 3 indicate that, independently of each effect, the CA is preferentially separated for the PEG-600-rich phase, as indicated by $K_{C A} \geq 5.6 \pm 0.6$ and $E E \geq 85.5 \pm 1.4 \%$. As previously demonstrated, due to its negative charge at neutral $\mathrm{pH}$, CA has a preference for the non-charged PEG-rich phase, in the PEG-600/NaPA-8000 system [6]. Since at neutral pH, the NaPA8000 is negatively charged due to the presence of carboxylic acid groups in the principal polymeric chain, it exerts a strong electrostatic repulsion over anionic solutes [20], which supports the preferential migration of the charged CA species for the PEG layer. From the data of Fig. 3 the effect of two parameters may be analyzed, namely the increase of the PEG-600 concentration and the influence of $[\mathrm{Ch}] \mathrm{Cl}$ presence as adjuvant. Although the increase of [Ch]Cl and PEG-600 concentration does not induce an inversion

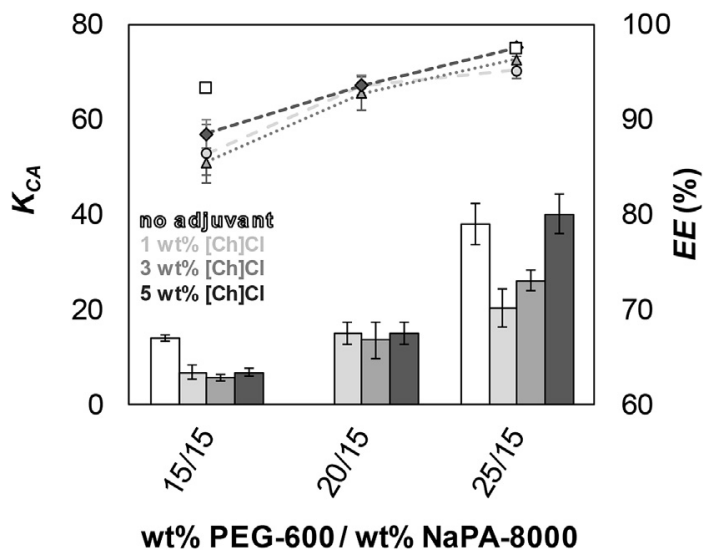

Fig. 3. Partition coefficients $\left(K_{C A} ; \square, \square, \square, \square\right)$ and extraction efficiencies (EE (\%); $\square, \bullet$, $\Delta$, ) of CA as a function of PEG-600/NaPA-8000/[Ch]Cl weight percentages of each ATPS, at $25^{\circ} \mathrm{C}$ and atmospheric pressure. The crescent gray gradient corresponds to the ATPS PEG-600/NaPA-8000 without adjuvant and with 1,3 and $5 \mathrm{wt} \%$ of $[\mathrm{Ch}] \mathrm{Cl}$ as adjuvant, respectively. Error bars represent standard deviation for three independent replicates. Dashed lines are only for guideline purposes. on the CA partitioning behavior, the control and adjustment of their concentrations can improve the CA extraction yields. A first analysis of the results according to the first parameter (PEG concentration) shows that an increase of PEG-600 from 15 to $25 \mathrm{wt} \%$ enlarges the recovery of CA for the PEG-rich phase (high $K_{C A}$ values). An increase in the PEG-600 composition allowed to recover over than $95 \%$ of CA in a single-step. On other hand, the addition of [Ch]Cl as adjuvant has a less pronounced effect on the drug separation. In general, [Ch] $\mathrm{Cl}$ reduces the CA affinity for PEG-rich phase [this effect is more pronounced for the small concentrations of PEG-600 (e.g. $15 \mathrm{wt} \%$ )], when compared with the PEG-600/NaPA-8000-based ATPS without adjuvant. As additive, and due to its low concentration in the overall system composition, $[\mathrm{Ch}] \mathrm{Cl}$ plays a secondary role on the partitioning of CA. However, when used the ATPS composed of 25 wt\% of PEG-600 and 15 wt\% of NaPA-8000, different compositions of $[\mathrm{Ch}] \mathrm{Cl}$ have influenced differently on the distribution of $\mathrm{CA}$ molecules between PEG- and NaPA-rich phases. The $K_{C A}$ has increased from $6.7 \pm 0.7$ with $1 \mathrm{wt} \%$ of [Ch]Cl to $40.0 \pm 4.2$ with $5 \mathrm{wt}$ $\%$ of $[\mathrm{Ch}] \mathrm{Cl}$, an increment of more than 5 -fold.

To understand the influence of each parameter evaluated, and the corresponding mechanisms behind the partitioning of CA, firstly, it is important to comprehend the nature of these polymeric PEG/NaPA systems. In this type of ATPS, the phase separation results from the incompatibility of water structures around the two polymers in equilibrium, where an increase of these incompatible polymer-water interactions leads to a greater tendency for the phase splitting [40]. The addition of salts' ions to aqueous polymeric mixtures re-arranges the water molecules distribution around the polymeric molecules, perturbing these polymermodified water structures. Depending on the ions' nature, these salts can infer a water-structure-"breaking" or -"making" behavior [40]. In addition, the formation of the two-phase regime in a PEG/ NaPA system is usually the result of the presence of the electrolyte ions which decreases the entropy of compartmentalization of the counter ions at the co-existing phases [58]. Thus, the influence of NaPA-8000 counter ions $\left(\mathrm{Na}^{+}\right)$is reduced with the addition of smaller ions to the system as adjuvants (as for example $[\mathrm{Ch}]^{+}$and $\mathrm{Cl}^{-}$), and then, they will "equilibrate" the ion concentration between both co-existing phases formed, thus facilitating the compartmentalization of the polyelectrolyte [58]. Herein, it seems that the reduction of CA partitioning for PEG-rich phase observed in most PEG-600/NaPA-8000-based ATPS using [Ch]Cl as adjuvant, outcomes from a combination of "salting-in" effects of $[\mathrm{Ch}] \mathrm{Cl}$ and the "equilibria" of the ions' gradient between both co-existing phases. In this sense, the CA electrostatic repulsion of NaPA-8000 was reduced, and the migration of CA molecules for the PEG-rich phase was decreased.

On other hand, the influence of PEG-600 concentration in the CA partitioning is quite more complex. The mechanistic phenomena for the formation of PEG/NaPA-based ATPS previously proposed were based on polymeric aqueous systems composed of PEG and NaPA polymers with high molecular weights. On the contrary, in this work a new type of PEG/NaPA-based ATPS formed using a small size PEG was studied. Herein, due to its low molecular weight, PEG600 exhibits a high hydrophilic nature (in comparison with large size polymers), since the ratio between $\mathrm{H}$-bond donor groups (the two terminal-OH groups of the polymeric chains) over the H-bond acceptor groups of the ethylene oxide units was enlarged, being the molecular mechanisms controlling the ATPS formation quite different. The partitioning capabilities of ATPS based in high molecular weight PEG/NaPA polymers have been evaluated for different types of molecules, namely CA [6], green fluorescent protein (GFP) [20], cytochrome $c$ protein [59], chloranilic acid dye [59], among others. A general analysis of previous partitioning results shows that anionic biomolecules, disregarding its hydrophilic/ 
hydrophobic nature, were always concentrated in the PEG-rich phase (similarly to that observed in this work). It seems evident that the electro-repulsive forces of NaPA-8000 over charged solutes is the main mechanism governing the solute preferential migration towards the PEG-rich phase, and that is non-dependent of the PEG size or solutes' nature. Since here it was pursued the separation of a highly hydrophilic solute (CA) using a polymeric ATPS composed of a quite hydrophilic PEG-600, it seems that a second type of interactions is also affecting the CA partitioning. The PEG concentration increase has led to an increment of the $K_{C A}$. As PEG-600 is quite hydrophilic, perhaps, the increased number of PEG molecules in the system "pulls out" more water molecules away from the NaPA-rich phase, changing the relative hydrophobicity of the coexisting phases of the system. Thus, due to CA intrinsic hydrophilicity, it seems that some favorable CA-water interactions may also contribute to the CA partitioning, in which a high number of CA molecules migrate with the water for the PEG-rich phase, increasing the $K_{C A}$ values. Although the relative hydrophobicity of the phases was not determined for this new type of systems, this second mechanism is supported by the comparison with our previous results obtained for the same solute and using PEG/NaPAbased ATPS composed of PEG-2000, PEG-4000, PEG-6000 or PEG10000 [6]. In these studies the $K_{C A}$ values obtained were similar for the different PEG polymers investigated, and in general, lower than the values attained in this work.

Summing up, the application of these PEG/NaPA-based ATPS in the extraction of commercial CA was demonstrated to be highly efficient. It was further demonstrated that the extraction and partition parameters can be improved by the increasing of the PEG composition.

\subsection{Recovery of CA from the fermented broth}

Based on the previous results obtained with the commercial source of CA, four systems were selected and further evaluated for the recovery and purification of CA directly from the fermented broth of $S$. clavuligerus, in a single-step process. The systems under study were: two PEG-600/NaPA-8000-based ATPS (one without adjuvant, and another with $5 \mathrm{wt} \%$ of $[\mathrm{Ch}] \mathrm{Cl})$; a PEG-600/[Ch]Clbased ATPS; and a PEG-550-OMe/[Ch]Cl-based ATPS. The four systems selected were studied as pre-purification platforms, in which it is expected their capacity to concentrate CA in just one phase with the contaminants (here evaluated as total protein content) being retained in the opposite layer. The results obtained are shown as Fig. 4 and Table S3 (Supporting Information).

The results depicted in Fig. 4 (and Table S3 from Supporting Information) seem to suggest that at least $44 \%$ of CA present in the fermented broth of $S$. clavuligerus $\left(>250 \mathrm{mg} \mathrm{L}^{-1}\right.$ ) was recovered in a single step, being the highest recovery yield $\left[\eta_{C A}=64.91 \pm 1.99(\%)\right]$ obtained with the polymeric PEG-600/NaPA-8000-based ATPS. Moreover, it is possible to conclude that the presence of $[\mathrm{Ch}] \mathrm{Cl}$ as adjuvant is not improving the results of recovery yield or purification $\left[\eta_{C A}=55.88 \pm 0.86(\%) ; P F=2.43 \pm 0.05\right]$ obtained for the conventional system $\left[\eta_{C A}=64.91 \pm 1.99(\%) ; P F=2.31 \pm 0.04\right]$, which follows the general conclusions obtained during the screening work carried with the commercial CA. Comparing both set of results of CA partition from fermented broth and the standard CA, when used the PEG-600/[Ch]Cl system an inversion of the solute partitioning for the $[\mathrm{Ch}] \mathrm{Cl}$-rich phase was observed (commercial CA was concentrated in the PEG-rich phase). This inversion may be explained by the presence of residual salts from the fermented medium, which are acting as "salting-out" agents towards CA. All systems have exhibited an ability to separate the CA from the contaminants proteins produced by the microorganism via fermentation, from 2-fold to 22-fold. Interestingly, the highest $P F$

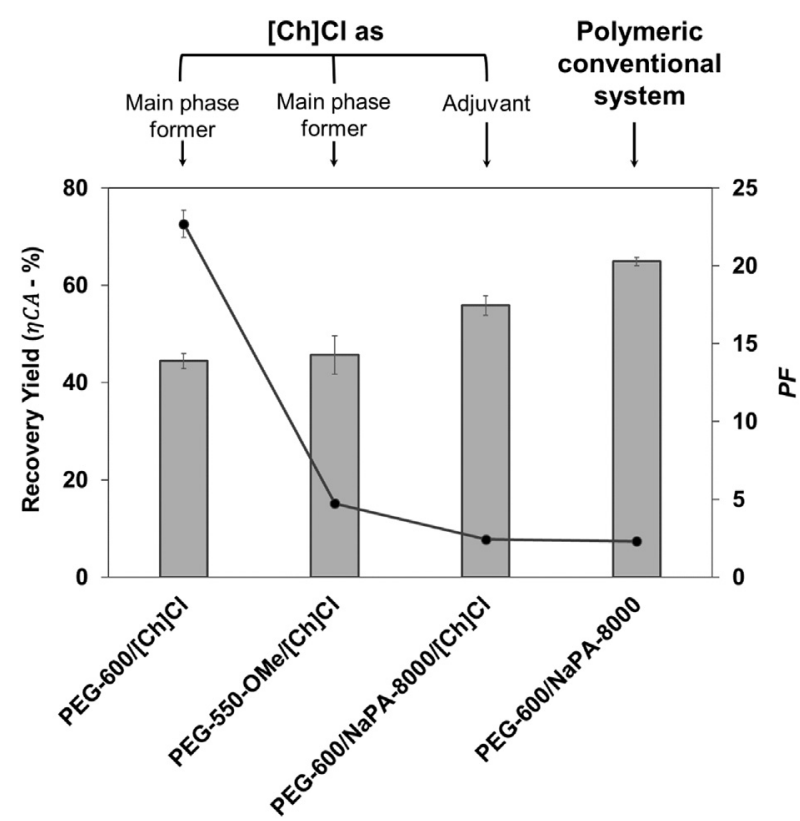

Fig. 4. Results of the recovery yields $\left[\eta_{C A}-\right.$ grey bar (\%)] and purification factor (PF $(\bullet)$ black line is only for guideline purposes) of CA produced via fermentation and obtained for different ATPS, at $25{ }^{\circ} \mathrm{C}$ and atmospheric pressure.

was attained with the ATPS with lowest recovery yield, the PEG$600 /[\mathrm{Ch}] \mathrm{Cl}$-based ATPS, where CA was purified more than 22-fold in the $[\mathrm{Ch}] \mathrm{Cl}$-rich phase $(P F=22.70 \pm 0.87)$. These results can be explained by the high structural instability of CA. In a complex fermented broth, where many proteins, cell-debris, and other impurities are present, not only the solute can be affected in terms of its preferential migration but can also suffer negative effects in terms of its potential degradation, imposing a decreasing in their recovery yields. As CA was directly extracted without any further stabilization procedure, the yields and respective $P F$ obtained in this work are quite significant.

Some authors have already developed different studies pursuing the same objective of to purify CA after its production via fermentation and using ATPS. The extractive fermentation of CA using ATPS composed of PEG and phosphate salts was investigated, where approximately 93\% of CA was recovered in the PEG-rich phase [53]. Moreover, mixed micellar systems have also demonstrated an ability to recover more than $90 \%$ of the CA again from fermented broth [4]. Hirata et al. have defined a combined separation process using precipitation steps and liquid-liquid extractions with yields between 30 and 73\% [43]. Unfortunately, these previous studies do not provide any further details about the CA purification $[4,43,53]$. Others have applied ionic exchange adsorption processes with CA recoveries from 60 to $100 \%$ and $P F$ around 1.5 [5,60]. Recoveries of CA between 49 and $99 \%$ and a $P F$ of 1.5 -fold were obtained when ATPS-based in phosphate/PEG systems were adopted [45]. Finally, ATPS composed of $20 \mathrm{wt} \%$ of PEG-4000, $20 \mathrm{wt}$ $\%$ of $\mathrm{NaPA}-8000$ and $6 \mathrm{wt} \%$ of $\mathrm{Na}_{2} \mathrm{SO}_{4}$ were reported to allow a $\eta_{C A}=55 \pm 5 \%$ and a $P F<1$ (proteins and CA are concentrated in the same phase) [6]. However, when compared our results with those already reported in literature, it is possible to conclude the highest efficiency of the system under study, in particular the system composed of PEG/[Ch]Cl-based ATPS, to purify CA $(P F=22.70 \pm 0.87)$. Actually, not only a more efficient process of purification of CA is here reported but also a simpler one. In addition, as the other CA recovery processes using ATPS $[6,7,45,50,53]$, the use of these systems is economic, biocompatible, easily to scale- 
up and may be further applied in a continuous chromatographic processing.

\section{Conclusions}

The partitioning and extraction of CA from commercial source and fermented broth of $S$. clavuligerus using biocompatible and stable [Ch]Cl-based and polymeric ATPS as effective liquid-liquid recovery and purification platforms were evaluated. Firstly, the CA stability studies have shown that CA remains stable in different $[\mathrm{Ch}] \mathrm{Cl}$ aqueous solutions. The systems composed of PEG-600 or PEG-550-OMe and $[\mathrm{Ch}] \mathrm{Cl}$ have demonstrated a similar partitioning aptitude to separate CA, where the CA was equally distributed between both phases in equilibrium and it was achieved up to $69 \%$ of extraction efficiencies. For the PEG-600/NaPA-8000 systems using [Ch]Cl as adjuvant, CA was preferentially separated into PEG-rich phase, and extraction efficiencies higher than $85 \%$ were attained. Moreover, the CA recovery studies from fermented broth have demonstrated at least 2-fold purification ability from the contaminant proteins. This purification factor was yielded in the system composed of PEG-600/[Ch]Cl, in which CA was purified in more than 22-fold towards the [ $\mathrm{Ch}] \mathrm{Cl}$-rich phase. These results support the use of the investigated systems as effective techniques to purify CA from fermented broth in a single partitioning step.

\section{Acknowledgements}

This work was developed under the scope of the project PADC 2014/16-I, financed by institutional funds of "Programa de Apoio ao Desenvolvimento Científico" from School of Pharmaceutical Sciences of Universidade Estadual Paulista (UNESP). Jorge F. B. Pereira acknowledges the support from FAPESP (São Paulo Research Foundation Brazil) through the Young Researcher Project (2014/ 16424-7). Camila Lopes acknowledges funding from CAPES. Valéria C. Santos-Ebinuma acknowledges the support from FAPESP through the Young Researcher Project (2014/01580-3). This work was developed within the scope of the project CICECO-Aveiro Institute of Materials, POCI-01-0145-FEDER-007679 (FCT Ref. UID/CTM/ 50011/2013), financed by national funds through the FCT/MEC and when appropriate co-financed by FEDER under the PT2020 Partnership Agreement. S.P.M. Ventura acknowledges the FCT/MCTES for a contract under Investigador FCT 2015 contract number IF/ $00402 / 2015$.

\section{Appendix A. Supplementary data}

Supplementary data related to this chapter can be found at http://dx.doi.org/10.1016/j.fluid.2017.07.005.

\section{References}

[1] P.S. Saudagar, S.A. Survase, R.S. Singhal, Clavulanic acid: a review, Biotechnol Adv. 26 (2008) 335-351, http://dx.doi.org/10.1016/j.biotechadv.2008.03.002.

[2] P.A. Bersanetti, R.M.R.G. Almeida, M. Barboza, M.L.G.C. Araújo, C.O. Hokka, Kinetic studies on clavulanic acid degradation, Biochem. Eng. J. 23 (2005) 31-36, http://dx.doi.org/10.1016/j.bej.2004.10.007.

[3] D.J. Kim, J.A. King, L. Zuccarelli, C.F. Ferris, G.A. Koppel, C.T. Snowdon, C.H. Ahn, Clavulanic acid: a competitive inhibitor of beta-lactamases with novel anxiolytic-like activity and minimal side effects, Pharmacol. Biochem. Behav. 93 (2009) 112-120, http://dx.doi.org/10.1016/j.pbb.2009.04.013.

[4] V.C. Santos, F.A. Hasmann, A. Converti, A. Pessoa, Liquid-liquid extraction by mixed micellar systems: a new approach for clavulanic acid recovery from fermented broth, Biochem. Eng. J. 56 (2011) 75-83, http://dx.doi.org/10.1016/ j.bej.2011.05.011.

[5] M. Barboza, R.M. Almeida, C.O. Hokka, Kinetic studies of clavulanic acid recovery by ion exchange chromatography, Bioseparation 10 (2001) 221-227, http://dx.doi.org/10.1023/A:1016365827265.

[6] J.F.B. Pereira, V.C. Santos, H.-O. Johansson, J.A.C. Teixeira, A. Pessoa Jr., A stable liquid-liquid extraction system for clavulanic acid using polymer-based aqueous two-phase systems, Sep. Purif. Technol. 98 (2012) 441-450, http:// dx.doi.org/10.1016/i.seppur.2012.08.008.

[7] M. Videira, M.R. Aires-barros, Liquid-liquid extraction of clavulanic acid using an aqueous two-phase system of polyethylene glycol and potassium phosphate, J. Chromatogr. 668 (1994) 237-240, http://dx.doi.org/10.1016/00219673(94)80113-4.

[8] D.B. Hirata, J.H.H.L. Oliveira, K.V. Leao, M.I. Rodrigues, a. G. Ferreira, M. Giulietti, M. Barboza, C.O. Hokka, Optimization of the precipitation of clavulanic acid from fermented broth using T-octylamine as intermediate, Braz. J. Chem. Eng. 30 (2013) 231-244, http://dx.doi.org/10.1590/S010466322013000200002.

[9] M. de S.C. Silva, V. de C. Santos-Ebinuma, A.M. Lopes, C. de O. Rangel-Yagui, Dextran sulfate/Triton $X$ two-phase micellasr systems as an alternative first purification step for clavulanic acid, Fluid Phase Equilib. 399 (2015) 80-86, http://dx.doi.org/10.1016/j.fluid.2015.04.026.

[10] M.G. Freire, A.F.M. Cláudio, J.M.M. Araújo, J.A.P. Coutinho, I.M. Marrucho, J.N.C. Lopes, L.P.N. Rebelo, Aqueous biphasic systems: a boost brought about by using ionic liquids, Chem. Soc. Rev. 41 (2012) 4966, http://dx.doi.org/ $10.1039 / \mathrm{c} 2 \mathrm{cs} 35151 \mathrm{j}$.

[11] J.F.B. Pereira, F. Vicente, V.C. Santos-Ebinuma, J.M. Araújo, A. Pessoa, M.G. Freire, J.A.P. Coutinho, Extraction of tetracycline from fermentation broth using aqueous two-phase systems composed of polyethylene glycol and cholinium-based salts, Process Biochem. 48 (2013) 716-722, http:// dx.doi.org/10.1016/j.procbio.2013.02.025.

[12] A. Wajid, F. Malik, S. Shafaat, S. Hussain, G. Parveen, S. Roohi, R. Mahmood, R.A. Channa, F.Y. Raja, M. Ismail, Effective extraction of cephalosporin C from whole fermentation broth of Acremonium chrysogenum utilizing aqueous two phase systems, Afr. J. Biotechnol. 11 (2012) 7973-7979, http://dx.doi.org/ 10.5897/AJB11.3443.

[13] Y.-C. Feng, W.-L. Li, F.-M. He, T.-T. Kong, X.-W. Huang, Z.-H. Gao, N.-H. Lu, H.L. Li, Aqueous two-phase system as an effective yool for purification of phenolic compounds from fig fruits ( Ficus carica L.), Sep. Sci. Technol. 50 (2015) 1785-1793, http://dx.doi.org/10.1080/01496395.2015.1014054.

[14] S. Li, C. He, H. Liu, K. Li, F. Liu, Ionic liquid-based aqueous two-phase system, a sample pretreatment procedure prior to high-performance liquid chromatography of opium alkaloids, J. Chromatogr. B. Biomed. Appl. 826 (2005) 58-62, http://dx.doi.org/10.1016/j.jchromb.2005.08.005.

[15] K.V.G. Barros, P.M. Souza, S.L. Cardoso, L.L. Borges, E.X.F. Filho, A.P. Junior, P.O. Magalhães, Extraction protease expressed by Penicillium fellutanum from Brazilian savanna using poly(ethylene glycol)/sodium polyacrylate/ $\mathrm{NaCl}$ aqueous two-phase systems, Biotechnol. Appl. Biochem. 62 (2015) 806-814, http://dx.doi.org/10.1002/bab.1337.

[16] A.W.F. Duarte, A.M. Lopes, J.V.D. Molino, A. Pessoa, L.D. Sette, Liquid-liquid extraction of lipase produced by psychrotrophic yeast Leucosporidium scottii L117 using aqueous two-phase systems, Sep. Purif. Technol. (2015) 1-11, http://dx.doi.org/10.1016/j.seppur.2015.10.001.

[17] E. Alvarez-Guerra, A. Irabien, Ionic Liquid-Based Three phase partitioning (ILTPP) for lactoferrin recovery, Sep. Sci. Technol. 49 (2014) 957-965, http:// dx.doi.org/10.1080/01496395.2013.878722.

[18] B.A. Andrews, J.A. Asenjo, Protein partitioning equilibrium between the aqueous poly(ethylene glycol) and salt phases and the solid protein phase in poly(ethylene glycol)-salt two-phase systems, J. Chromatogr. B Biomed. Appl. 685 (1996) 15-20, http://dx.doi.org/10.1016/0378-4347(96)00134-X.

[19] B. Mokhtarani, R. Karimzadeh, M.H. Amini, S.D. Manesh, Partitioning of Ciprofloxacin in aqueous two-phase system of poly(ethylene glycol) and sodium sulphate, Biochem. Eng. J. 38 (2008) 241-247, http://dx.doi.org/10.1016/ j.bej.2007.07.009.

[20] H.O. Johansson, M. Ishii, M. Minaguti, E. Feitosa, T.C.V. Penna, A. Pessoa, Separation and partitioning of Green Fluorescent Protein from Escherichia coli homogenate in poly(ethylene glycol)/sodium-poly(acrylate) aqueous twophase systems, Sep. Purif. Technol. 62 (2008) 166-174, http://dx.doi.org/ 10.1016/j.seppur.2008.01.017.

[21] L.D. Lario, M.L. Pellegrini, J.F.B. Pereira, L.D. Sette, A. Pessoa, Liquid-liquid extraction of protease from cold-adapted yeast Rhodotorula mucilaginosa L7 using biocompatible and biodegradable aqueous two-phase systems, Sep. Sci. Technol. $51 \quad$ (2016) 57-67, http://dx.doi.org/10.1080/ 01496395.2015.1080276.

[22] R.L. Souza, S.P.M. Ventura, C.M.F. Soares, J.A.P. Coutinho, Á.S. Lima, Lipase purification using ionic liquids as adjuvants in aqueous two-phase systems, Green Chem. 17 (2015) 3026-3034, http://dx.doi.org/10.1039/C5GC00262A.

[23] P.L. Santos, L.N.S. Santos, S.P.M. Ventura, R.L. de Souza, J.A.P. Coutinho, C.M.F. Soares, Á.S. Lima, Recovery of capsaicin from Capsicum Frutescens by applying aqueous two-phase systems based on acetonitrile and xholiniumbased ionic liquids, Chem. Eng. Res. Des. 112 (2016) 103-112, http:// dx.doi.org/10.1016/j.cherd.2016.02.031.

[24] C.P. Song, R.N. Ramanan, R. Vijayaraghavan, D.R. MacFarlane, E.-S. Chan, C.W. Ooi, Green, Aqueous two-phase systems based on cholinium aminoate ionic liquids with tunable hydrophobicity and charge density, ACS Sustain. Chem. Eng. 3 (2015) 3291-3298, http://dx.doi.org/10.1021/ acssuschemeng.5b00881.

[25] Z. Li, X. Liu, Y. Pei, J. Wang, M. He, Design of environmentally friendly ionic liquid aqueous two-phase systems for the efficient and high activity extraction of proteins, Green Chem. 14 (2012) 2941, http://dx.doi.org/10.1039/ c2gc35890e.

[26] M.V. Quental, M. Caban, M.M. Pereira, P. Stepnowski, J.A.P. Coutinho, 
M.G. Freire, Enhanced extraction of proteins using cholinium-based ionic liquids as phase-forming components of aqueous biphasic systems, Biotechnol. J. 10 (2015) 1457-1466, http://dx.doi.org/10.1002/biot.201500003.

[27] R.F.F. de Araujo, T.S. Porto, D.B.G. Martins, R.F. Dutra, A.L.F. Porto, Partitioning of lactate dehydrogenase from bovine heart crude extract by polyethylene glycol-citrate aqueous two-phase systems, Fluid Phase Equilib. 301 (2011) 46-50, http://dx.doi.org/10.1016/j.fluid.2010.11.016.

[28] R.L. Souza, R.A. Lima, J.A.P. Coutinho, C.M.F. Soares, Á.S. Lima, Aqueous twophase systems based on cholinium salts and tetrahydrofuran and their use for lipase purification, Sep. Purif. Technol. 155 (2014) 118-126, http:// dx.doi.org/10.1016/j.seppur.2015.05.021.

[29] K.A. Zeisel, S.H. da Costa, Choline: an essential nutrient for public health, Nutr. Rev. $67 \quad$ (2009) 615-623, http://dx.doi.org/10.1111/j.17534887.2009.00246.x.

[30] J.K. Blusztajn, Developmental neuroscience: enhanced: choline, a vital amine, Science (80-. ) 281 (1998) 794-795, http://dx.doi.org/10.1126/ science.281.5378.794.

[31] H. Zhao, G.A. Baker, Ionic liquids and deep eutectic solvents for biodiesel synthesis: a review, J. Chem. Technol. Biotechnol. 88 (2013) 3-12, http:// dx.doi.org/10.1002/jctb.3935.

[32] S. Shahriari, L.C. Tomé, J.M.M. Araújo, L.P.N. Rebelo, J.A.P. Coutinho, I.M. Marrucho, M.G. Freire, Aqueous biphasic systems: a benign route using cholinium-based ionic liquids, RSC Adv. 3 (2013) 1835-1843, http:// dx.doi.org/10.1039/c2ra22972b.

[33] M. Petkovic, J.L. Ferguson, H.Q.N. Gunaratne, R. Ferreira, M.C. Leitão, K.R. Seddon, L.P.N. Rebelo, C.S. Pereira, Novel biocompatible cholinium-based ionic liquids-toxicity and biodegradability, Green Chem. 12 (2010) 643, http://dx.doi.org/10.1039/b922247b.

[34] R.S. Boethling, E. Sommer, D. DiFiore, Designing small molecules for biodegradability, Chem. Rev. 107 (2007) 2207-2227, http://dx.doi.org/10.1021/ cr050952t.

[35] S.P.M. Ventura, F.A. e Silva, A.M.M. Gonçalves, J.L. Pereira, F. Gonçalves, J.A.P. Coutinho, Ecotoxicity analysis of cholinium-based ionic liquids to Vibrio fischeri marine bacteria, Ecotoxicol. Environ. Saf. 102 (2014) 48-54, http:// dx.doi.org/10.1016/j.ecoenv.2014.01.003.

[36] J.I. Santos, A.M.M. Goncalves, J.L. Pereira, B.F.H.T. Figueiredo, F.A. e Silva, J.A.P. Coutinho, S.P.M. Ventura, F. Goncalves, Environmental safety of cholinium-based ionic liquids: assessing structure-ecotoxicity relationships, Green Chem. 17 (2015) 4657-4668, http://dx.doi.org/10.1039/C5GC01129A.

[37] J.F.B. Pereira, L.P.N. Rebelo, R.D. Rogers, J.A.P. Coutinho, M.G. Freire, Combining ionic liquids and polyethylene glycols to boost the hydrophobic-hydrophilic range of aqueous biphasic systems, Phys. Chem. Chem. Phys. 15 (2013) 19580-19583, http://dx.doi.org/10.1039/С3CP53701C.

[38] G.M. Powell, Handbook of Water Soluble Gums and Resins, McGraw-Hill, New York, 1980.

[39] D.A. Herold, K. Keil, D.E. Bruns, Oxidation of polyethylene glycols by alcohol dehydrogenase, Biochem. Pharmacol. 38 (1989) 73-76, http://dx.doi.org/ 10.1016/0006-2952(89)90151-2.

[40] V. Gupta, S. Nath, S. Chand, Role of water structure on phase separation in polyelectrolyte-polyethyleneglycol based aqueous two-phase systems, Polym. Guildf. 43 (2002) 3387-3390, http://dx.doi.org/10.1016/S00323861(02)00117-9.

[41] S. Saravanan, J.A. Reena, J.R. Rao, T. Murugesan, B.U. Nair, Phase equilibrium compositions, densities, and viscosities of aqueous two-phase poly (ethylene glycol) + poly (acrylic acid) system at various temperatures, J. Chem. Eng. Data 51 (2006) 1246-1249, http://dx.doi.org/10.1021/je050543u.

[42] H.O. Johansson, F.M. Magaldi, E. Feitosa, A. Pessoa, Protein partitioning in poly (ethylene glycol)/sodium polyacrylate aqueous two-phase systems, J. Chromatogr. A 1178 (2008) 145-153, http://dx.doi.org/10.1016/ j.chroma.2007.11.071.

[43] D.B. Hirata, J.H.H.L. Oliveira, K.V. Leão, M.I. Rodrigues, A.G. Ferreira, M. Giulietti, M. Barboza, C.O. Hokka, Precipitation of clavulanic acid from fermentation broth with potassium 2-ethyl hexanoate salt, Sep. Purif. Technol 66 (2009) 598-605, http://dx.doi.org/10.1016/j.seppur.2009.01.010.

[44] C.S. da Silva, M.F. Cuel, V.O. Barreto, W.H. Kwong, C.O. Hokka, M. Barboza, Separation of clavulanic acid from fermented broth of amino acids by an aqueous two-phase system and ion-exchange adsorption, N. Biotechnol. 29 (2012) 428-431, http://dx.doi.org/10.1016/j.nbt.2011.05.012.

[45] C.S. Silva, E. Bovarotti, M.I. Rodrigues, C.O. Hokka, M. Barboza, Evaluation of the effects of the parameters involved in the purification of clavulanic acid from fermentation broth by aqueous two-phase systems, Bioprocess Biosyst. Eng. 32 (2009) 625-632, http://dx.doi.org/10.1007/s00449-008-0285-6.

[46] J.C. Teodoro, A. Baptista-Neto, M.L.G.C. Araujo, C.O. Hokka, A.C. Badino, Influence of glycerol and ornithine feeding on clavulanic acid production by streptomyces clavuligerus, Braz. J. Chem. Eng. 27 (2010) 499-506, http:// dx.doi.org/10.1590/S0104-66322010000400001.

[47] A.E. Bird, J.M. Bellis, B.C. Gasson, Spectrophotometric assay of clavulanic acid by reaction with lmidazole, Analyst 107 (1982) 1241-1245, http://dx.doi.org/ 10.1039/AN9820701241.

[48] A. Jerzsele, G. Nagy, The stability of amoxicillin trihydrate and potassium clavulanate combination in aqueous solutions, Acta Vet. Hung 57 (2009) 485-493, http://dx.doi.org/10.1556/AVet.57.2009.4.3.

[49] V.C. Santos, J.F.B. Pereira, R.B. Haga, C.O. Rangel-Yagui, J.A.C. Teixeira A. Converti, A. Pessoa, Stability of clavulanic acid under variable pH, ionic strength and temperature conditions. A new kinetic approach, Biochem. Eng. J. 45 (2009) 89-93, http://dx.doi.org/10.1016/j.bej.2009.02.013.

[50] M.N. Carneiro-da-Cunha, K.P.S. Souza, A.M.O. Mota, J.A. Teixeira, C.S. Porto, T.S. Porto, A.L.F. Porto, Stability of clavulanic acid in PEG/citrate and liquid-liquid extraction in aqueous two-phase system, Fluid Phase Equilib. 375 (2014) 104-109, http://dx.doi.org/10.1016/j.fluid.2014.04.036.

[51] New YorkK.J. Laidler (Ed.), Chemical Kinetics, 1950.

[52] J.F.B. Pereira, K.A. Kurnia, M.G. Freire, J.A.P. Coutinho, R.D. Rogers, Controlling the formation of ionic-liquid-based aqueous biphasic systems by changing the hydrogen-bonding ability of polyethylene glycol end groups, ChemPhysChem 16 (2015) 2219-2225, http://dx.doi.org/10.1002/cphc.201500146.

[53] D.A. Viana Marques, A. Pessoa-Júnior, J.L. Lima-Filho, A Converti, P. Perego, A.L.F. Porto, Extractive fermentation of clavulanic acid by Streptomyces DAUFPE 3060 using aqueous two-phase system, Biotechnol. Prog. 27 (2011) 95-103, http://dx.doi.org/10.1002/btpr.526.

[54] G.H. Hakimelahi, K.S. Shia, C. Xue, S. Hakimelahi, A.A. Moosavi-Movahedi, A.A. Saboury, A. Khalafi-Nezhad, M.N. Soltani-Rad, V. Osyetrov, K.-P. Wang, J.H. Liao, F.-T. Luo, Design, synthesis, and biological evaluation of a series of $\beta$ lactam-based prodrugs, Bioorg. Med. Chem. 10 (2002) 3489-3498, http:/ dx.doi.org/10.1016/S0968-0896(02)00256-0.

[55] Y. Zhang, P.S. Cremer, Interactions between macromolecules and ions: the Hofmeister series, Curr. Opin. Chem. Biol. 10 (2006) 658-663, http:// dx.doi.org/10.1016/j.cbpa.2006.09.020.

[56] S. Shahriari, C.M.S.S. Neves, M.G. Freire, J.A.P. Coutinho, Role of the hofmeister series in the formation of ionic-liquid-based, J. Phys. Chem. B 116 (2012) 7252-7258, http://dx.doi.org/10.1021/jp300874u.

[57] J.F.B. Pereira, A. Magri, M.V. Quental, M. Gonzalez-Miquel, M.G. Freire, J.A.P. Coutinho, Alkaloids as alternative probes to characterize the relative hydrophobicity of aqueous biphasic systems, ACS Sustain. Chem. Eng. 4 (2016) 1512-1520, http://dx.doi.org/10.1021/acssuschemeng.5b01466.

[58] H.-O. Johansson, E. Feitosa, A.P. Junior, Phase diagrams of the aqueous twophase systems of poly(ethylene glycol)/sodium polyacrylate/salts, Polymers 3 (2011) 587, http://dx.doi.org/10.3390/polym3010587.

[59] J.H.P.M. Santos, F.A.E. Silva, J.A.P. Coutinho, S.P.M. Ventura, A. Pessoa, Ionic liquids as a novel class of electrolytes in polymeric aqueous biphasic systems, Process Biochem. 50 (2015) 661-668, http://dx.doi.org/10.1016/ j.procbio.2015.02.001.

[60] R.M.R.G. Almeida, M. Barboza, C.O. Hokka, Continuous clavulanic acid adsorption process, Appl. Biochem. Biotechnol. $105-108$ (2003) 867-879 http://dx.doi.org/10.1385/ABAB:108:1-3:867. 